\title{
Détermination à l'aide d'un modèle récepteur des zones sources à l'origine des concentrations mesurées dans les précipitations collectées en trois sites du réseau MERA (France) Source receptor modeling using precipitation data collected at three rural French sampling stations of MERA network
}

\author{
P. Coddeville, A. Charron, H. Plaisance et J. C. Galloo
}

Volume 15, numéro hors-série, 2002

URI : https://id.erudit.org/iderudit/705493ar

DOI : https://doi.org/10.7202/705493ar

Aller au sommaire du numéro

Éditeur(s)

Université du Québec - INRS-Eau, Terre et Environnement (INRS-ETE)

ISSN

0992-7158 (imprimé)

1718-8598 (numérique)

Découvrir la revue

Citer cet article

Coddeville, P., Charron, A., Plaisance, H. \& Galloo, J. C. (2002). Détermination à l'aide d'un modèle récepteur des zones sources à l'origine des concentrations mesurées dans les précipitations collectées en trois sites du réseau MERA (France). Revue des sciences de l'eau / Journal of Water Science, 15, 205-227. https://doi.org/10.7202/705493ar

\section{Résumé de l'article}

Ces travaux s'inscrivent dans le cadre du programme national de MEsure des Retombées Atmosphériques (MERA). Ils portent sur la recherche de l'origine des précipitations collectées entre 1997 et 1999 dans trois (Morvan, Iraty, Le Casset) des onze stations du réseau MERA localisées en différents points du territoire français. Deux méthodes statistiques ont été utilisées dans cette étude. Les régions à l'origine des fortes concentrations mesurées au site récepteur ont d'abord été déterminées à l'aide d'un modèle (méthode de Seibert) combinant les mesures réalisées sur site et les rétrotrajectoires de masses d'air puis, dans un second temps les différents profils de transport atmosphérique, leur fréquence et concentrations associées ont été évaluées à l'aide d'une classification par Nuées Dynamiques (méthode K-means/distance Euclidienne simple) des rétrotrajectoires de masses d'air. Le test de Kruskal-Wallis a été utilisé pour vérifier si les médianes des concentrations associées à chaque classe sont statistiquement différentes. L'étude réalisée à Iraty (Pyrénées) et au Casset (Alpes) a montré que ces deux stations sont influencées différemment du Morvan. Plus exactement, ces deux sites ne sont pas, ou pratiquement pas, influencés par les zones d'Europe centrale ou du Nord-Ouest fortement émettrices de $\mathrm{SO} 2$, de $\mathrm{NOx}$ et de $\mathrm{NH}_{3}$. Seul le $\mathrm{pH}$ des précipitations collectées à Iraty semble dépendre des émissions de SO2 et de NOx d'une de ces zones. Iraty et le Casset sont très influencées par les émissions anthropiques et par les poussières d'origine terrestres en provenance d'Afrique du Nord. Néanmoins, les niveaux de concentrations mesurés dans les flux en provenance d'Afrique du Nord sont similaires pour Iraty, le Casset et le Morvan (sauf en ions calcium, pour lequel le Casset et Iraty montrent de fortes concentrations). Une autre région européenne peut influencer les niveaux en composés acidifiants mesurés au Casset, il s'agit de l'Italie et de la zone localisée au niveau de l'ex-Yougoslavie. Mais, les niveaux de concentrations qui en résultent sont faibles par rapport à ceux mesurés dans certains flux arrivant au Morvan.
Ce document est protégé par la loi sur le droit d'auteur. L’utilisation des services d'Érudit (y compris la reproduction) est assujettie à sa politique d'utilisation que vous pouvez consulter en ligne.

https://apropos.erudit.org/fr/usagers/politique-dutilisation/ 


\title{
Détermination à l'aide d'un modèle récepteur des zones sources à l'origine des concentrations mesurées dans les précipitations collectées en trois sites du réseau MERA (France)
}

\author{
Source receptor modeling using precipitation data \\ collected at three rural French sampling stations \\ of MERA network
}

\author{
P. CODDEVILLE, A. CHARRON, H. PLAISANCE, J.C. GALLOO
}

\section{SUMMARY}

The chemistry of precipitation in France was examined using data from the French atmospheric deposition network (MERA). In order to examine the source-receptor relationships responsible for acid rain at three background sites in France, a receptor-oriented model was applied to the precipitation data collected from 1997 to 1999 . This methodology combined precipitation and chemical data with air parcel backward trajectories to establish concentration field maps of likely contributing sources. Then, a clustering technique using partitioning methods (K-means/Euclidian distance) was performed to backward trajectories and the distributions of mixing samples associated with backward trajectories in each cluster were compared. The KruskalWallis test was used to verify that the concentration medians associated with each cluster were statistically significant. The results of this study demonstrated that two stations (Iraty and le Casset) were not influenced by the same sources as Morvan. Specifically, these sites were less influenced by high emissions from Central or Northwestern Europe when compared to Morvan. Only the pH seemed under the influence of $\mathrm{SO}_{2}$ and $\mathrm{NO}_{x}$ emissions from one of these areas. Iraty and Le casset are very influenced by anthropogenic emissions and the crustal sources around the Mediterranean Basin and North Africa. Other European areas (e.g. Italy) can influence the concentrations recorded at Le Casset but the levels of concentration are lower than those measured at Morvan.

École des Mines de Douai, Département chimie et environnement, 841 rue Charles Bourseul, 59508 Douai, France.

* Correspondance. E-mail : coddeville@ensm-douai.fr

** Les commentaires seront reçus jusqu'au 30 avril 2003. 
This paper represents a complete statistical analysis of wet-only deposition chemistry data for three stations (Iraty, Le Casset and Morvan). Two statistical methods were used in this study. In order to examine the source-receptor relationships responsible for acid rain at these three background sites in France, a receptor-oriented model was applied to the precipitation data collected from 1997 to 1999 . This methodology combined chemical data with air parcel backward trajectories to establish concentration field maps of likely contributing sources. This receptor-oriented model was developed by Seibert and it assumes that if a trajectory endpoint falls in a grid cell $(i, j)$, the air mass is assumed to collect components emitted in this cell and once the components are incorporated, they are transported along the trajectory to the receptor site. This model doesn't take into account the atmospheric diffusion and the removal mechanisms occurring during the trajectory from the sources to the receptor. Finally, a concentration field map for the selected species was calculated taking into account all grid cells. For mapping, the grid cells counting fewer than $\mathbf{1 0}$ endpoints were not taken in consideration because the confidence of their results was considered too low. The role of three-dimensional backward trajectories is fundamental, so we used three different information sources: the French Institute of Meteorology, MétéoFrance; the British Atmospheric Data Centre (BADC); and the Atmospheric Environment Service Long Range Transport model of Air Pollution (AESLRTAP), Canada. These trajectory models were compared for different chemical species. All data were projected in the EMEP grid $(150 \times 150 \mathrm{~km})$ for establishment of the concentration field map. A clustering technique by partitioning methods (K-means/Euclidian distance) was performed on backward trajectories and the distributions of mixing samples associated with backward trajectories in each cluster were compared. The Kruskal-Wallis test was used to verify that the median concentrations associated with each cluster were statistically significant.

The results of this study for Morvan determined five classes of backward trajectories associated with the precipitation collected at this station located in the centre of France. The fluxes from SW and WSW sectors contribute for $52 \%$ of events, while the fluxes of $\mathrm{NW}$ and $\mathrm{E}$ contribute for $31 \%$ of events but are mainly responsible for high concentrations of sulphates, nitrates, ammonium and hydronium ion. Regions found to be responsible for rain events coincide with European regions known for their high anthropogenic emissions of $\mathrm{SO}_{2}$ and $\mathrm{NO}_{\mathrm{x}}$ (Great Britain, North of France, Belgium, The Netherlands and the North of sea).

The results for Iraty (South of France) yielded five classes of backward trajectories associated with the precipitation collected in this station. The fluxes from W sectors (NNW, NW, W and WSW) were responsible for $71 \%$ of events, while the flux of $S$ (low wind) was responsible for $29 \%$ of events but is mainly responsible for high concentrations of sulphates, nitrates, ammonium and calcium. High concentrations of hydronium ion were identified in the NNW sector.

The results for Le Casset (East region and mountainous) gave four classes of backward trajectories associated with the precipitation collected in this station. The fluxes from W and WSW sectors were responsible for $35 \%$ of events, while the flux of SSW was responsible for $43 \%$ and the flux from the SE was responsible for $22 \%$ of events. This last sector was mainly responsible for high concentrations of sulphates, nitrates, ammonium and calcium. The concentrations measured at this station were low. Regions found to be responsible for rain events coincide with southern and eastern areas known for their high anthropogenic emissions of $\mathrm{SO}_{2}$ and $\mathrm{NO}_{x}$ (north Africa, northern Italy, Yugoslavia).

All these results demonstrate that the Iraty and Le Casset stations were not influenced by the same sources as Morvan. Specifically, these sites were less 
influenced by the high emissions from central or northwestern Europe than Morvan. Only the measurement of $\mathrm{pH}$ seemed to be under the influence of $\mathrm{SO}_{2}$ and $\mathrm{NO}_{x}$ emissions of one of these areas. Iraty and Le Casset were very influenced by the anthropogenic emissions and the crustal sources around the Mediterranean Basin and North Africa. Other European areas (e.g., Italy) can influence the concentrations recorded at Le Casset but the levels were lower than those measured at Morvan. A relation between sulphates, nitrates and ammonium was identified for Morvan and Le Casset. This observation suggests that aerosol transport of $\mathrm{NH}_{4} \mathrm{HSO}_{4},\left(\mathrm{NH}_{4}\right)_{2} \mathrm{SO}_{4}$ and $\mathrm{NH}_{4} \mathrm{NO}_{3}$ is occurring.

Key-words: acid rain, rural sampling stations, source receptor backword trajectory, receptor model.

RÉSUMÉ

Ces travaux s'inscrivent dans le cadre du programme national de Mesure des retombées atmosphériques (MERA). Ils portent sur la recherche de l'origine des précipitations collectées entre 1997 et 1999 dans trois (Morvan, Iraty, Le Casset) des onze stations du réseau MERA localisées en différents points du territoire français. Deux méthodes statistiques ont été utilisées dans cette étude. Les régions à l'origine des fortes concentrations mesurées au site récepteur ont d'abord été déterminées à l'aide d'un modèle (méthode de Seibert) combinant les mesures réalisées sur site et les rétrotrajectoires de masses d'air puis, dans un second temps les différents profils de transport atmosphérique, leur fréquence et concentrations associées ont été évaluées à l'aide d'une classification par Nuées dynamiques (méthode K-means/distance Euclidienne simple) des rétrotrajectoires de masses d'air. Le test de KruskalWallis a été utilisé pour vérifier si les médianes des concentrations associées à chaque classe sont statistiquement différentes. L'étude réalisée à Iraty (Pyrénées) et au Casset (Alpes) a montré que ces deux stations sont influencées différemment du Morvan. Plus exactement, ces deux sites ne sont pas, ou pratiquement pas, influencés par les zones d'Europe centrale ou du NordOuest fortement émettrices de $\mathrm{SO}_{2}$, de $\mathrm{NO}_{x}$ et de $\mathrm{NH}_{3}$. Seul le $\mathrm{pH}$ des précipitations collectées à Iraty semble dépendre des émissions de $\mathrm{SO}_{2}$ et de $\mathrm{NO}_{x}$ d'une de ces zones. Iraty et le Casset sont très influencées par les émissions anthropiques et par les poussières d'origine terrestres en provenance d'Afrique du Nord. Néanmoins, les niveaux de concentrations mesurés dans les flux en provenance d'Afrique du Nord sont similaires pour Iraty, le Casset et le Morvan (sauf en ions calcium, pour lequel le Casset et Iraty montrent de fortes concentrations). Une autre région européenne peut influencer les niveaux en composés acidifiants mesurés au Casset, il s'agit de l'Italie et de la zone localisée au niveau de l'ex-Yougoslavie. Mais, les niveaux de concentrations qui en résultent sont faibles par rapport à ceux mesurés dans certains flux arrivant au Morvan.

Mots clés : précipitations acides, zones rurales, transport à longues distances, rétrotrajectoires, modele récepteur.

\section{1 - INTRODUCTION}

Le réseau de Mesure des retombées atmosphériques (MERA) a été institué en 1984 par le ministère de l'Environnement. Sa mise en place répond aux engagements pris par la France en matière de surveillance dans le cadre de la 
convention de Genève sur le transport transfrontalier de la pollution atmosphérique. Les objectifs du programme MERA sont donc liés à ceux du programme européen EMEP'1. Les points les plus importants du programme sont la surveillance et la caractérisation de la pollution atmosphérique en zones rurales non influencées localement ainsi que l'identification des régions sources pouvant être à l'origine des polluants acidifiants et l'évaluation de l'impact du transport à longues distances. Le réseau MERA comporte aujourd'hui 11 stations réparties sur l'ensemble du territoire français dont 8 sont incluses dans le réseau EMEP. Dans ce cadre, les précipitations sont collectées quotidiennement dans l'ensemble des stations du réseau depuis maintenant une dizaine d'années.

Les rétrotrajectoires constituent un outil très efficace pour déterminer les sources à l'origine des polluants atmosphériques mesurés sur des sites de fond. Des ensembles importants de rétrotrajectoires sont interprétés en utilisant des méthodes statistiques. Ces méthodes se classent en trois groupes :

1. les analyses " par secteur " (ou " Flow climatology ») (e.g. MILLER et al., 1993) ;

2. les classifications automatiques, hiérarchiques (e.g. MOODY et SAMSON, 1989) ou par Nuées dynamiques (e.g. SIROIS et BOTTENHEIM, 1995);

3. les méthodes statistiques qui permettent l'établissement de la cartographie des sources probables sous forme de champs de probabilité (ASHBAUGH et al., 1985) ou de concentration (SEIBERT et al., 1994 ; STOHL, 1996).

Cette étude porte sur la détermination des origines géographiques des précipitations mesurées en zones rurales et notamment des composés acidifiants, sur l'évaluation de l'impact du transport à longues distances et sur l'examen des paramètres susceptibles de jouer un rôle sur les variations de ces composés.

Les objectifs fixés sont les suivants:

- la définition des zones émettrices de polluants acidifiants influençant différentes zones rurales françaises ;

- la compréhension des différences observées entre les différentes zones rurales étudiées. En particulier, nous nous sommes intéressés à l'impact de la « climatologie » des masses d'air sur chaque site étudié.

\section{2 - DONNÉES CHOISIES}

L'étude a porté sur l'ensemble des composés acidifiants mesurés dans le réseau MERA, et notamment certains ions mesurés dans les précipitations potentiellement traceurs de composés acidifiants. Les ions $\mathrm{SO}_{4}^{2-}$ et $\mathrm{NO}_{3}^{-}$sont

1. Cooperative programme for the monitoring and evaluation of long range air pollutants in Europe. 
des traceurs des émissions anthropiques de $\mathrm{SO}_{2}$ et de $\mathrm{NO}_{\mathrm{x}}$. Ils sont connus comme étant les principaux gaz responsables de l'acidité des précipitations (GALLOWAY et al., 1976 ; SEINFELD et PANDIS, 1996). L'ion $\mathrm{NH}_{4}^{+}$, acide conjugué de l'ammoniac $\mathrm{NH}_{3}$, est aussi un composé important dans le cadre d'une recherche de sources de composés acidifiants. II contribue à l'acidification des écosystèmes par l'intermédiaire du processus de nitrification dans les sols (FANGMEIER et al., 1994). L'ion $\mathrm{Ca}^{2+}$ provient essentiellement du carbonate de calcium $\mathrm{CaCO}_{3}$ (LOYE-PILOT et MORELLI, 1988), d'origine terrigène et de l'oxyde de calcium CaO (MARQUARDT et IHLE, 1988), issu de la combustion du charbon. Le calcium, tout comme l'ammoniac $\mathrm{NH}_{3}$ (SEINFELD et PANDIS, 1996 ; MIHALOPOULOS et al., 1997) a un rôle important dans les processus de neutralisation de l'acidité des précipitations (MUNGER, 1982).

\section{3 - SITES CHOISIS}

Les sites retenus pour cette étude sont représentés sur la carte 1 et caractérisent différentes influences météorologiques possibles.

\subsection{Morvan}

Ce site est localisé dans le Parc naturel régional du Morvan, dans le département de la Côte d'Or, à $85 \mathrm{~km}$ à l'Ouest de Dijon (longitude : 4,05 E, latitude: $47,16 \mathrm{~N}$ ). La station est à une position sommitale à une altitude de 620 mètres. Le tableau 1 rassemble les caractéristiques des données étudiées.

Tableau 1 Caractéristiques des données utilisées pour les stations d'Iraty, du Morvan et du Casset.

Table 1 Data characteristics used for Iraty, Morvan and Casset stations ( $N$ is the number of samples and $P$ the altitude of backward trajectories at the receptor site).

\begin{tabular}{|c|c|c|c|c|}
\hline \multicolumn{2}{|c|}{} & Iraty & Morvan & Le Casset \\
\hline Échantillons & Dates & $1997-1999$ & $1992-1995$ & $1997-$ Juil. 2000 \\
& $N$ & 399 & 386 & $138^{*}$ \\
Rétro & Origine & BADC $^{*}$ & A. Sirois, SEA** & BADC* $^{*}$ \\
trajectoires & $N$ & 438 & 386 & 164 \\
& $P$ & $800 \mathrm{hPa}$ & $800 \mathrm{hPa}$ & $780 \mathrm{hPa}$ \\
\hline
\end{tabular}

$N$ est le nombre d'échantillons et $P$ l'altitude de calcul au site récepteur.

*British atmospheric data centre ; ${ }^{\star \star}$ Service de l'environnement atmosphérique, Québec. 


\subsection{Iraty}

Ce site est localisé dans les Pyrénées-Atlantiques, sur le plateau d'Iraty (longitude : $1,08 \mathrm{E}$; latitude : 43,03 N) à une altitude de 1300 mètres. Il se situe à une trentaine de kilomètre de l'océan Atlantique. Le tableau 1 regroupe les caractéristiques des données utilisées.

\subsection{Le Casset}

Le Casset est situé dans le Parc national des Écrins, dans le département des Hautes-Alpes (longitude : 6,31 E ; latitude : 44,59 N). La station est localisée dans la vallée de la Guisane, à une altitude de 1750 mètres. Tous les échantillons correspondant à des événements de neige n'ont pas été pris en compte en raison de l'incertitude sur l'heure de l'événement. Pour cette raison, seuls les échantillons de pluies ont été inclus dans cette étude. Le tableau 1 regroupe les caractéristiques des données utilisées. Pour pallier au faible nombre d'échantillons pris en compte dans l'étude, et du fait que la période de collecte ne peut être élargie, nous avons décidé d'utiliser une technique itérative de rééchantillonnage avec remise (bootstrapping) permettant d'augmenter le nombre de données disponibles. Cette technique consiste à sélectionner de façon aléatoire un certain nombre de données (concentrations associées à une rétrotrajectoire) de l'ensemble initial et à introduire ces données dans le calcul du champ de concentrations. Ce calcul sera répété $n$ fois et comparé aux résultats obtenus avec les données initiales.

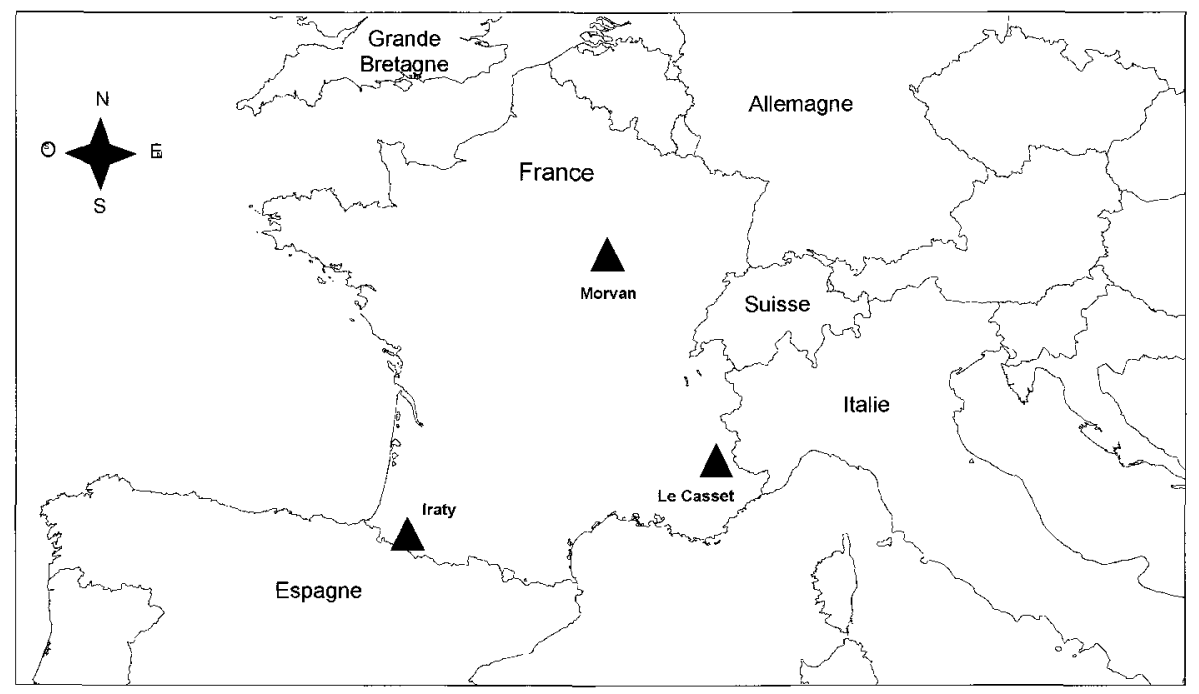

Carte 1

Situation géographique des stations d'études.

Location of the studied sites. 


\section{4 - MÉTHODOLOGIES UTILISÉES}

Les cartes de sources probables ont été calculées à l'aide de la méthode de SEIBERT et al. (1994). Des rétrotrajectoires de masses d'air tridimensionnelles calculées au BADC (British atmospheric data center) et au AES-LRTAP (Atmospheric environment service-long range transport model of air pollution) (VOLDNER et al., 1981) définissent le trajet emprunté par les masses d'air avant leur arrivée au site récepteur. Chaque échantillon de pluie journalière est associé à 4 rétrotrajectoires calculées au site récepteur pour les heures de 12 hUT, 18 hUT, 0 hUT et 6 hUT. Les rétrotrajectoires sont représentées dans la grille définie par EMEP à l'aide d'une projection stéréographique polaire vraie à la latitude $60^{\circ} \mathrm{N}$. Cette méthodologie suppose que l'ensemble des zones géographiques survolé par les masses d'air est potentiellement responsable des concentrations mesurées au site récepteur. La qualité des résultats obtenus dépend du nombre de rétrotrajectoires. Chaque point de rétrotrajectoires est associé à la concentration observée au site récepteur. Un point de trajectoire correspond à la position géographique de la masse d'air avant son arrivée au site récepteur. Les points sont projetés dans la grille EMEP de maillage 150 sur $150 \mathrm{~km}$. Des concentrations moyennes sont attribuées à chaque maille du domaine. Les mailles auxquelles moins de 10 points de trajectoires sont affectés sont retirées en raison de leur faible signification statistique. Pour plus de détails sur ces méthodologies se référer à CHARRON (1999).

La technique du bootstrapping a été appliquée aux données de pluies du Casset de façon à augmenter le nombre de données disponibles pour le calcul des cartes de sources probables.

La classification par Nuées dynamiques K-means a permis le classement des rétrotrajectoires par classe de rétrotrajectoires en se basant sur leur provenance et la vitesse des vents. Les concentrations associées à chaque classe sont ensuite représentées à l'aide de boîtes à moustaches.

\section{5 - ORIGINES DES POLLUANTS ACIDIFIANTS MESURÉS DANS LES PLUIES}

\subsection{Morvan}

Les cartes des concentrations modélisées en sulfates, nitrates et ammonium sont présentées sur les figures 1 à 3 .

La figure 4 présente les résultats de la classification par Nuées dynamiques appliquée aux rétrotrajectoires associées aux précipitations collectées au Morvan entre 1992 et 1995.

La classification par Nuées dynamiques nous a permis de définir 5 classes pour le Morvan dont 4 correspondant à des vents en provenance de l'Ouest. Ces résultats sont en accord avec la climatologie de la France : le régime des vents d'ouest est responsable de la plupart des précipitations en France 


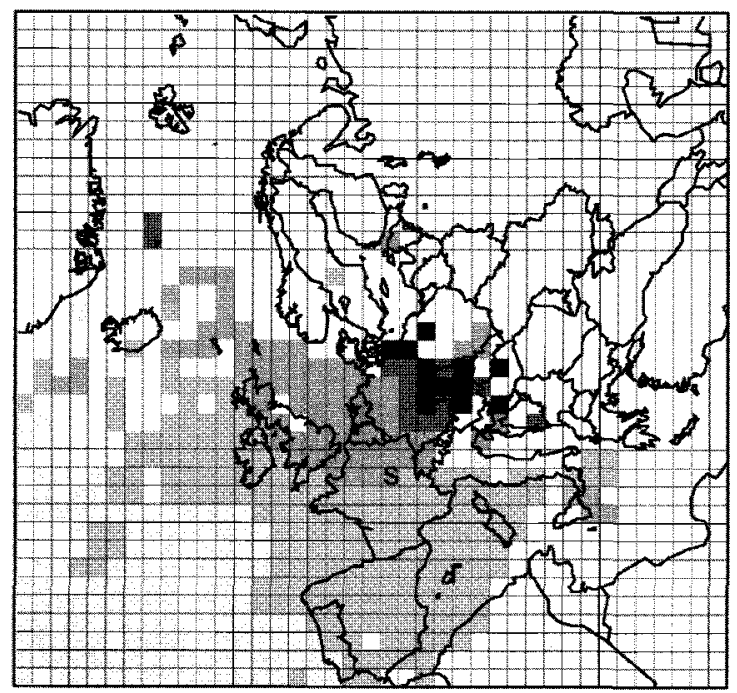

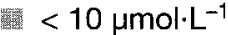

$10-20 \mu \mathrm{mol} \cdot \mathrm{L}^{-1}$

国 $20-40 \mu \mathrm{mol} \cdot \mathrm{L}^{-1}$

- $40-50 \mu \mathrm{mol} \cdot \mathrm{L}^{-1}$

- $>50 \mu \mathrm{mol} \cdot \mathrm{L}^{-1}$

Figure 1 Concentrations modélisées de nss- $\mathrm{SO}_{4}^{2-}$ calculées avec la méthode de SEIBERT et al. et les trajectoires du SEA, Québec.

Average Morvan nss- $\mathrm{SO}_{4}^{2-}$ concentrations in precipitation according to the origins of air masses

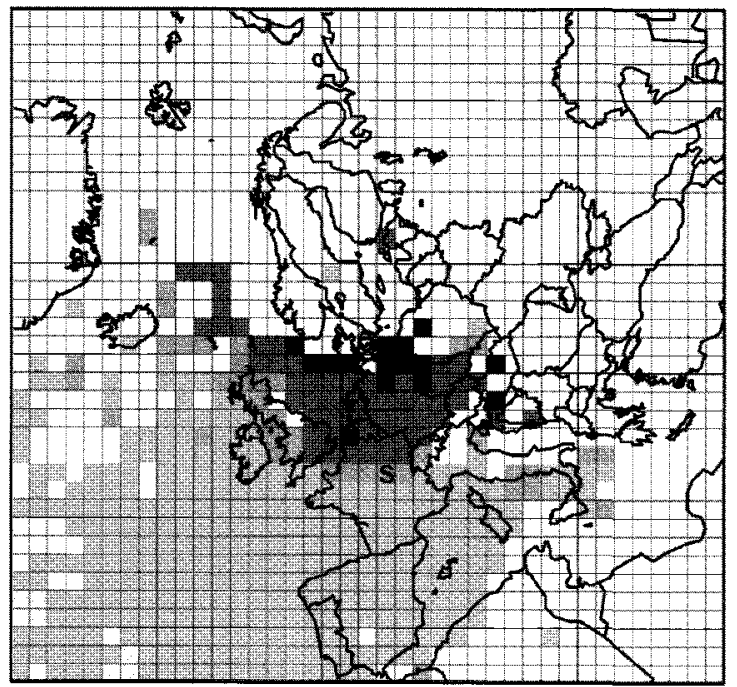

酸 $<10 \mu \mathrm{mol} \cdot \mathrm{L}^{-1}$

$10-30 \mu \mathrm{mol} \cdot \mathrm{L}^{-1}$

露 $30-40 \mu \mathrm{mol} \cdot \mathrm{L}^{-1}$

- $40-60 \mu \mathrm{mol} \cdot \mathrm{L}^{-1}$

a $>60 \mu \mathrm{mol} \cdot \mathrm{L}^{-1}$

Figure 2 Concentrations modélisées de $\mathrm{NO}_{3}^{-}$calculées avec la méthode de SEIBERT et al. et les trajectoires du SEA, Québec.

Average Morvan $\mathrm{NO}_{3}^{-}$concentrations in precipitation according to the origins of air masses.

(ESTIENNE et GODARD, 1970). Les courants de SO et de OSO sont les plus importants au Morvan, ils représentent à eux deux 51,5\% des événements pluvieux. Les vents de NO ne représentent que 16,3\% des événements. Nous 


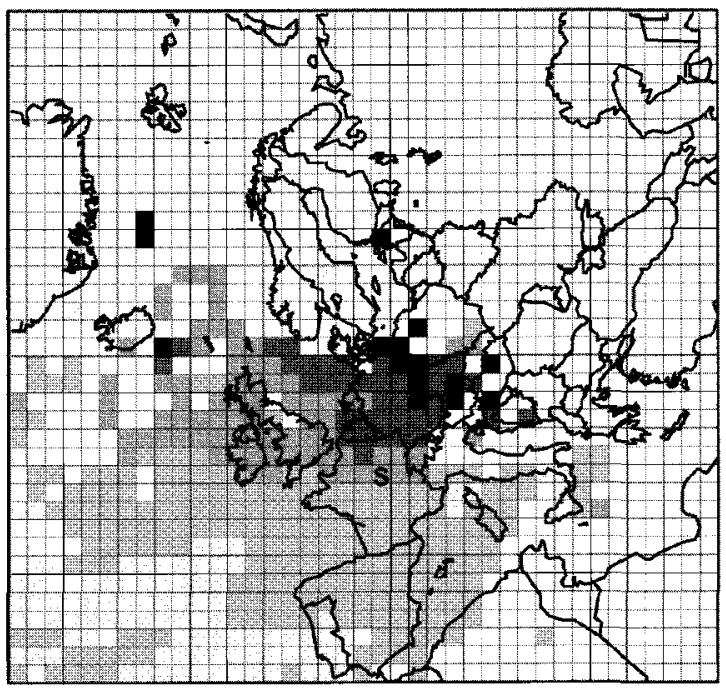

㘍 $<50 \mu \mathrm{mol} \cdot \mathrm{L}^{-1}$

$50-80 \mu \mathrm{mol} \cdot \mathrm{L}^{-1}$

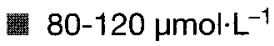

- $120-150 \mu \mathrm{mol} \cdot \mathrm{L}^{-1}$

च $>150 \mu \mathrm{mol} \cdot \mathrm{L}^{-1}$

Figure 3 Concentrations modélisées en ammonium $\mathrm{NH}_{4}^{+}$calculées avec la méthode de SEIBERT et al. et les trajectoires du SEA, Québec.

Average Morvan $\mathrm{NH}_{4}^{+}$concentrations in precipitation according to the origins of air masses.

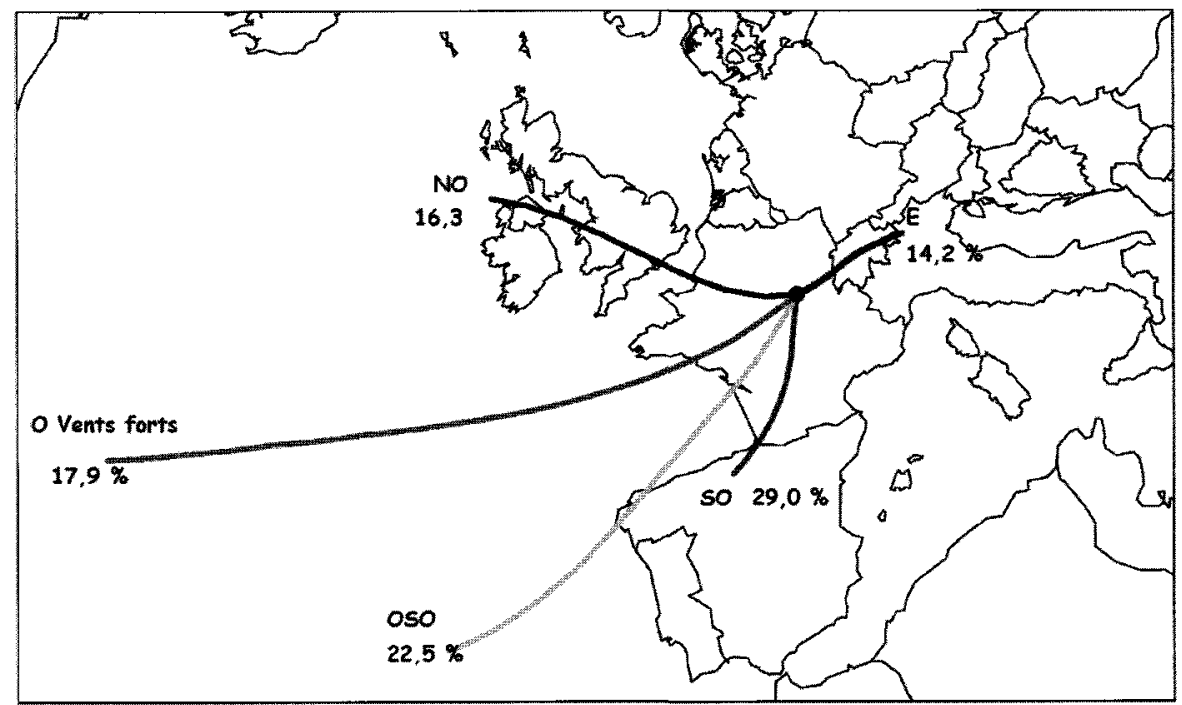

Figure 4

Centres finaux des classes des rétrotrajectoires associées aux événements pluvieux collectés au Morvan pendant la période 1992-1995.

$K-M e a n s$ clustering analysis associated with the backtrajectories of rainfall events at Morvan (1992-1995).

avons observé, en accord avec ESTIENNE et GODARD (1970) qu'ils sont plus fréquents au printemps. Enfin les flux d'Est ne sont à l'origine que de 14,2\% des événements pluvieux. Ces flux sont provoqués par la présence d'une zone 


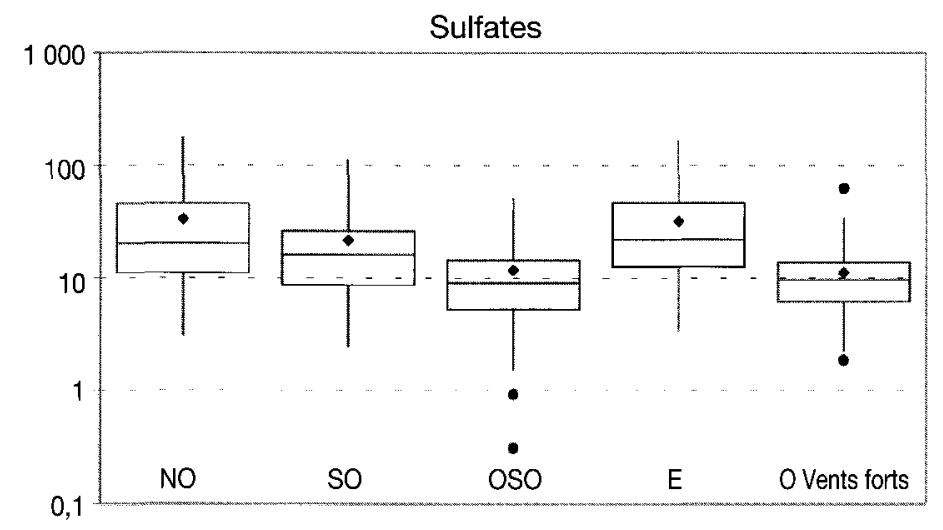

Figure 5 Boîtes à moustaches des concentrations en ions sulfates en $\mu \mathrm{mole} \cdot \mathrm{L}^{-1}$ associées à chaque classe de secteur prédominant.

Box and whiskers plots for nss-SO $\mathrm{SO}_{4}^{2-}$ concentrations $\left(\mu \mathrm{mol} \cdot \mathrm{L}^{-1}\right)$ in precipitation at Morvan and associated with each class of trajectory analyse.

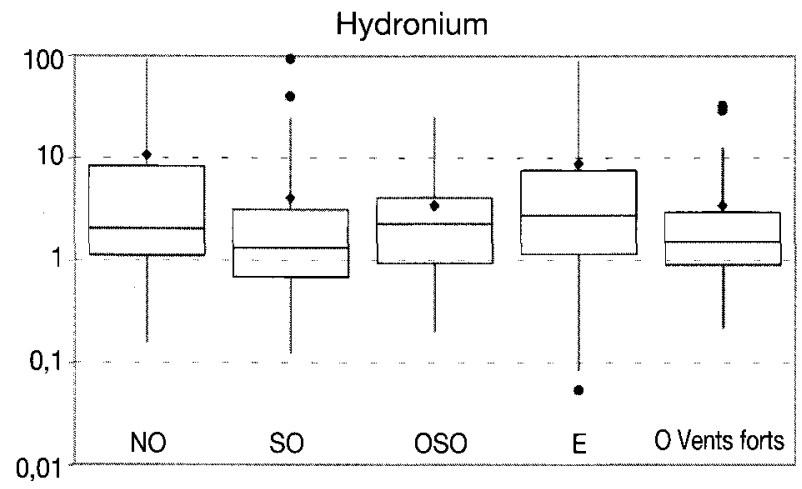

Figure 6 Boîtes à moustaches des concentrations en ions $\mathrm{H}^{+}$en $\mu$ mole $\cdot \mathrm{L}^{-1}$ associées à chaque classe de secteur prédominant.

Box and whiskers plots for $\mathrm{H}^{+}$concentrations $\left(\mu \mathrm{mol} \cdot \mathrm{L}^{-1}\right)$ in precipitation at Morvan and associated with each class of trajectory analyse.

dépressionnaire en Méditerranée et de zones de hautes pressions sur l'océan Atlantique et sur l'Europe Centrale (ESTIENNE et GODARD, 1970). Ces deux dernières directions de provenance minoritaires sont à l'origine des précipitations les plus chargées en sulfates, nitrates, ammonium et hydronium (figures 5 et 6). La classification a permis de faire ressortir l'importance de la direction NO sur le budget des sulfates, nitrates et ammonium dans les précipitations collectées au Morvan. Cette direction de provenance correspond aux îles Britanniques et probablement aussi au nord de la France, à la Belgique, aux Pays-Bas et à la Mer du Nord. La plus grande difficulté à cerner cette zone émettrice à l'aide de la méthode de SEIBERT et al. est peut-être la conséquence d'une plus grande incertitude sur le tracé des rétrotrajectoires au niveau des zones océaniques. 
L'application d'une Analyse en composantes principales (ACP) avec rotation varimax aux concentrations ioniques associées aux différents secteurs de provenance confirme le fort lien entre les ions sulfates, nitrates et ammonium. Ces trois ions sont quelquefois associés avec les ions calcium (secteur NO et OSO). Une composante marine composée de 2 ou 3 ions est observable pour tous les secteurs, même pour le secteur Est. Enfin, les concentrations en ions hydronium ne semblent être liées à aucun ion pour l'ensemble des secteurs, sauf pour le secteur SO pour lequel ils sont faiblement corrélés aux ions nitrates.

\subsection{Iraty}

Les trois cartes des figures 7 à 9 présentent les résultats obtenus avec la méthode de SEIBERT et al. pour les ions nss- $\mathrm{SO}_{4}^{2-}, \mathrm{NO}_{3}^{-}$et $\mathrm{NH}_{4}^{+}$. Nous avons retranché la part des concentrations de sulfates provenant du sel de mer (LOSNO et al., 1991). Comme pour le Morvan, l'océan Atlantique s'est montré comme premier contribuant en sodium à Iraty.

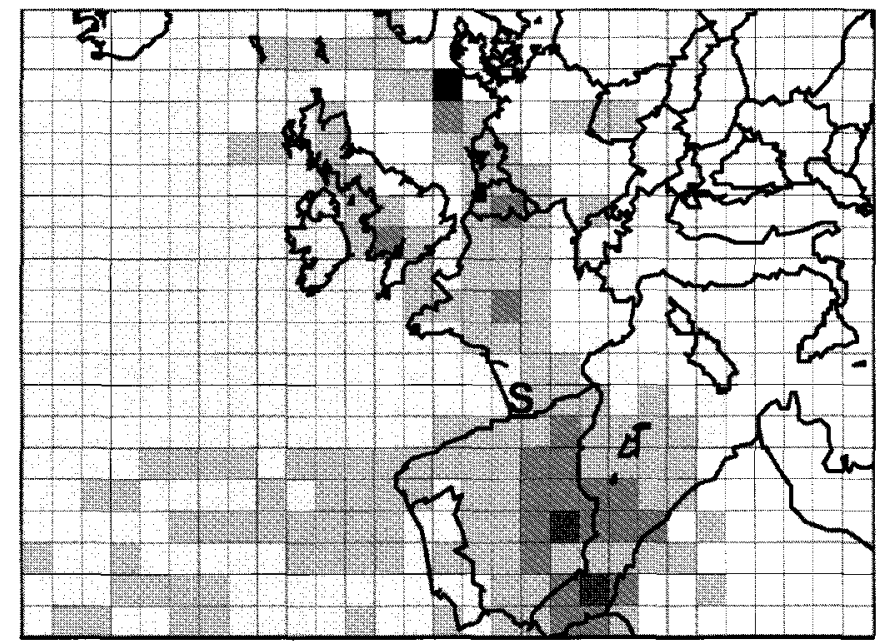
䗡 $<10 \mu \mathrm{mol} \cdot \mathrm{L}^{-1}$
10-20 $\mu \mathrm{mol} \cdot \mathrm{L}^{-1}$

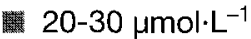
$30-40 \mu \mathrm{mol} \cdot \mathrm{L}^{-1}$
a $>40 \mu \mathrm{mol} \cdot \mathrm{L}^{-1}$

Figure 7

Concentrations modélisées de sulfates non marins mesurés dans les pluies collectées à Iraty (1997-1999).

Average Iraty nss- $\mathrm{SO}_{4}^{2-}$ concentrations in precipitation (1997-1999) according to the origins of air masses.

De plus fortes concentrations en ions nss- $\mathrm{SO}_{4}^{2-}, \mathrm{NO}_{3}^{-}$et $\mathrm{NH}_{4}^{+}$sont attribuées au sud-ouest de l'Espagne et au nord de l'Afrique mais aussi à quelques cellules isolées dans le nord de la France, en Belgique, aux Pays-Bas, et en Angleterre. D'après les cartes des émissions fournies par EMEP, certaines de ces zones correspondent bien à des zones fortement émettrices de $\mathrm{SO}_{2}$ et de $\mathrm{NO}_{\mathrm{x}}$. En revanche, le sud de l'Espagne ne montre pas d'émissions de $\mathrm{SO}_{2}$ et de $\mathrm{NO}_{x}$ notables (mis à part le détroit de Gibraltar, du fait de sa circulation maritime). Nous ne possédons pas de données sur les émissions du nord de l'Afrique. L'ensemble des concentrations en ammonium attribuées aux différentes mailles du domaine est très faible par rapport à celles attribuées aux 


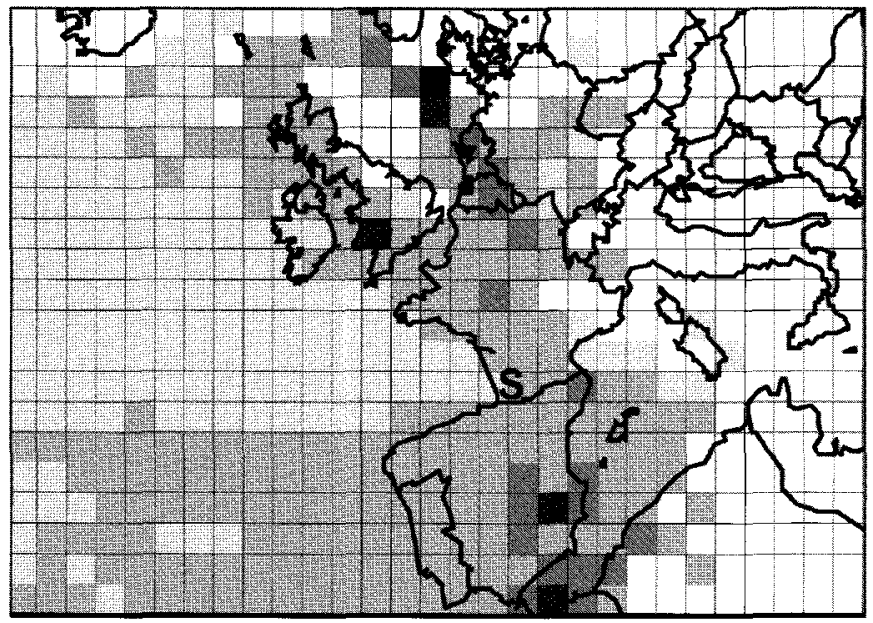

䌯 $<10 \mu \mathrm{mol} \cdot \mathrm{L}^{-1}$

$10-30 \mu \mathrm{mol} \cdot \mathrm{L}^{-1}$

麗 $30-40 \mu \mathrm{mol} \cdot \mathrm{L}^{-1}$

- $40-60 \mu \mathrm{mol} \cdot \mathrm{L}^{-1}$

a $>60 \mu \mathrm{mol} \cdot \mathrm{L}^{-1}$

Figure 8 Concentrations modélisées de nitrates mesurés dans les pluies collectées à Iraty (1997-1999).

Average Iraty $\mathrm{NO}_{3}^{-}$concentrations in precipitation (1997-1999) according to the origins of air masses.

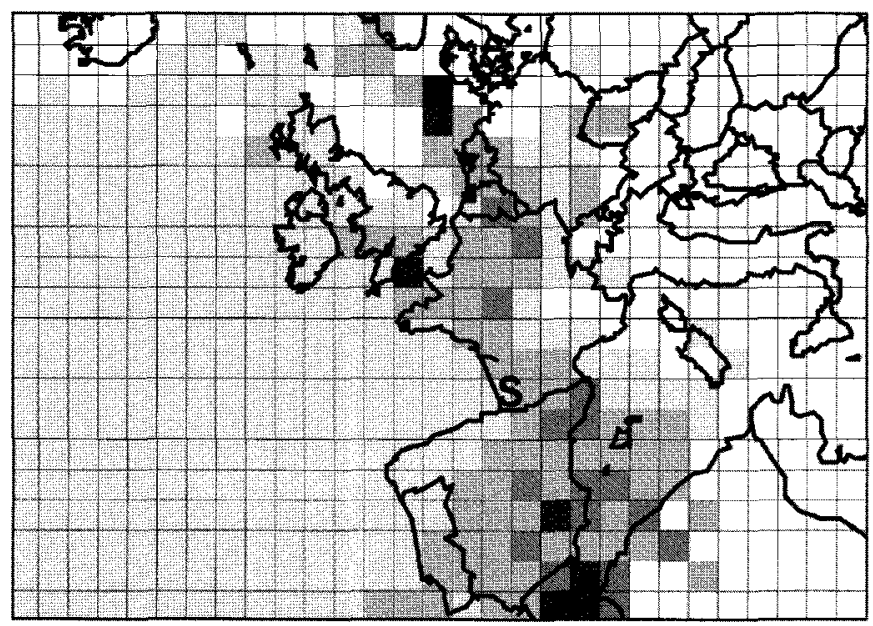

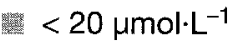

20-40 $\mu \mathrm{mol} \cdot \mathrm{L}^{-1}$

霹 $40-50 \mu \mathrm{mol} \cdot \mathrm{L}^{-1}$

- $50-80 \mu \mathrm{mo} \cdot \mathrm{L}^{-1}$

$>80 \mu \mathrm{mol} \cdot \mathrm{L}^{-1}$

Figure 9

Concentrations modélisées d'ammonium mesurées dans les pluies collectées à Iraty (1997-1999).

Average Iraty $\mathrm{NH}_{4}^{+}$concentrations in precipitation (1997-1999) according to the origins of air masses.

mailles de la carte établie pour le Morvan. Les origines géographiques de l'ammonium semblent mieux coïncider avec celles des ions sulfates et nitrates qu'avec les zones fortement émettrices.

La carte des concentrations modélisées de calcium non marin (figure 10) montre que le nord de l'Afrique contribue aux plus fortes concentrations en calcium à Iraty. Ce résultat est en accord avec la carte des émissions de calcium 


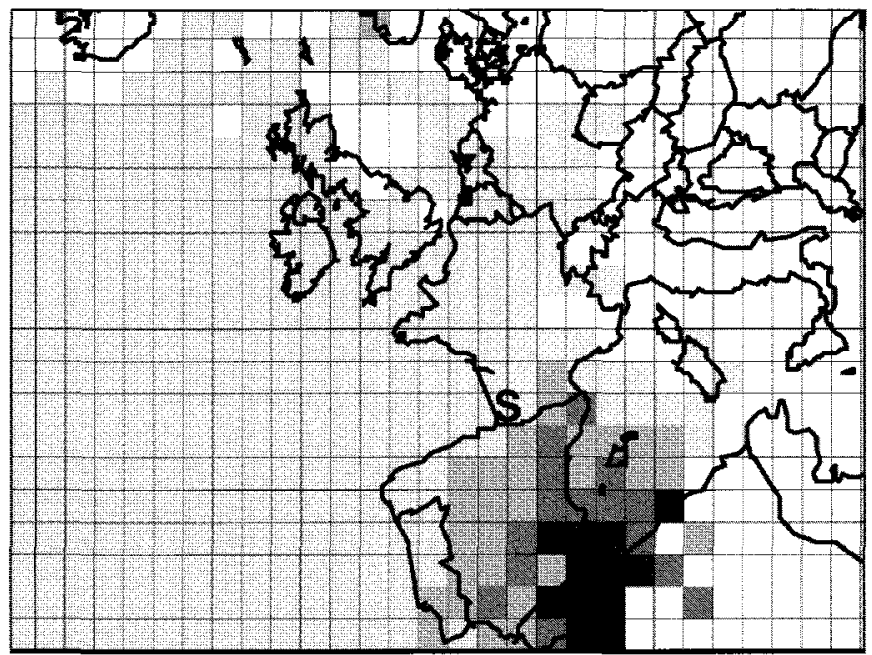

黨 $<10 \mu \mathrm{mol} \cdot \mathrm{L}^{-1}$

政 $10-20 \mu \mathrm{mol} \cdot \mathrm{L}^{-1}$

20-30 $\mu \mathrm{mol} \cdot \mathrm{L}^{-1}$

- $30-40 \mu \mathrm{mol} \cdot \mathrm{L}^{-1}$

- $>40 \mu \mathrm{mol} \cdot \mathrm{L}^{-1}$

Figure 10 Concentrations modélisées de calcium non marin mesurées dans les pluies collectées à Iraty (1997-1999).

Average Iraty nss-Ca+ concentrations in precipitation (1997-1999) according to the origins of air masses.

établie par LEE et al. (1999). L'Espagne est montrée comme second contribuant en calcium. Deux hypothèses peuvent expliquer ce résultat. La mise en suspension de poussières d'origines terrestres dans les zones désertiques ou semi désertiques d'Espagne constitue la première hypothèse. La seconde hypothèse est l'effet du passage quasi systématique des masses d'air en provenance d'Afrique sur l'Espagne conduisant à une surestimation de sa contribution.

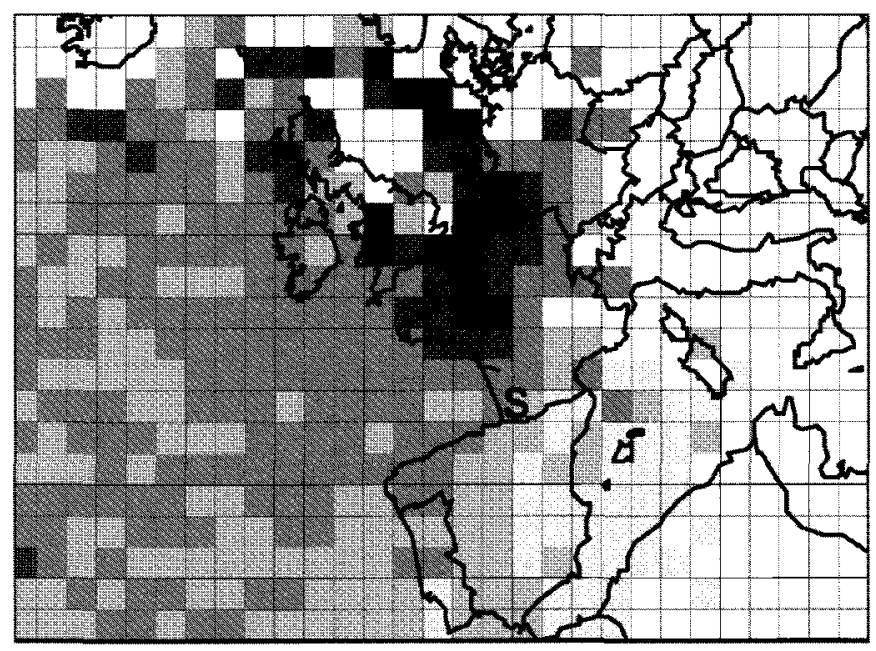

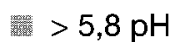

$5,8-5,4 \mathrm{pH}$

露 $5,4-5,0 \mathrm{pH}$

를 $5,0-4,8 \mathrm{pH}$

- $<4,8 \mathrm{pH}$

Figure 11 Concentrations modélisées en $\mathrm{H}^{+}$(exprimé en $\mathrm{pH}$ ) mesurés dans les pluies collectées à Iraty (1997-1999).

Average Iraty $\mathrm{H}^{+}(\mathrm{pH})$ concentrations in precipitation (1997-1999) according to the origins of air masses. 
La carte du $\mathrm{pH}$ (figure 11) identifie une large zone comme responsable des précipitations de $\mathrm{pH}$ inférieur à 4,8. Cette zone correspond à l'Angleterre, au nord de la France, à la Belgique et à la Mer du Nord. Elle coïncide donc avec des régions fortement émettrices de dioxyde de soufre et d'oxydes d'azote. Cette zone n'est cependant pas associée à de très fortes concentrations en sulfates et en nitrates, traceurs des composés acides $\mathrm{H}_{2} \mathrm{SO}_{4}$ et $\mathrm{HNO}_{3}$. Elle n'est pas associée non plus à de fortes concentrations en ammonium et calcium, traceurs de composés neutralisants. Ces pluies seraient donc acides car elles n'auraient pas été neutralisées. De fortes valeurs en $\mathrm{pH}$ supérieures à 5,8 sont associées avec le nord de l'Afrique et l'Espagne, régions montrant de fortes concentrations en calcium. Ce résultat indique l'impact de la calcite $\mathrm{CaCO}_{3}$. Cette zone montre aussi de fortes concentrations en sulfates et nitrates indiquant que des composés acides ont peut-être été neutralisés par $\mathrm{CaCO}_{3}$. Le transport atmosphérique sur de longues distances de $\mathrm{CaCO}_{3}$ est maintenant bien connu (COUDÉ-GAUSSEN et al., 1987 ; SEQUEIRA, 1993). L'impact de ces poussières terrigènes sur le $\mathrm{pH}$ a déjà été observé en France (LOYE-PILOT et MORELLI, 1988 ; LOSNO et al., 1991 ; MAUPETIT et DELMAS, 1994).

Cinq classes de rétrotrajectoires ressortent de la classification par Nuées dynamiques (figure 12) dont 4 correspondent à des vents en provenance de secteur Ouest (NNO, NO, O et OSO). Ces 4 classes constituent $71,3 \%$ des événements, ce qui montre l'importance des vents d'Ouest sur ce site localisé à proximité de l'océan Atlantique. La cinquième classe correspond à des vents en provenance du sud (Espagne).

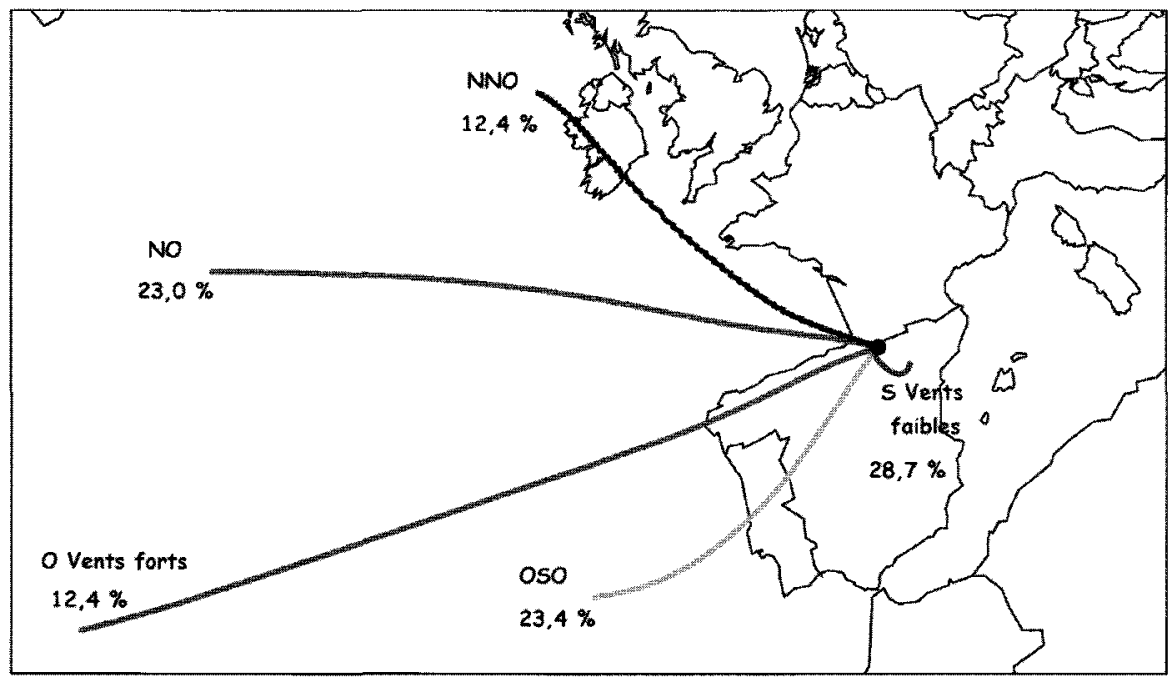

Figure 12

Centres finaux des classes de rétrotrajectoires associées aux événements pluvieux collectés à Iraty pour la période 1997-1999.

$K$-Means clustering analysis associated with the backtrajectories of rainfall events at Iraty (1997-1999).

Les concentrations des ions sulfates et hydronium associées aux 5 secteurs de provenance sont représentées à l'aide de boîtes à moustaches sur les figures 13 et 14. Le secteur Sud constitue le premier contribuant en ions sul- 


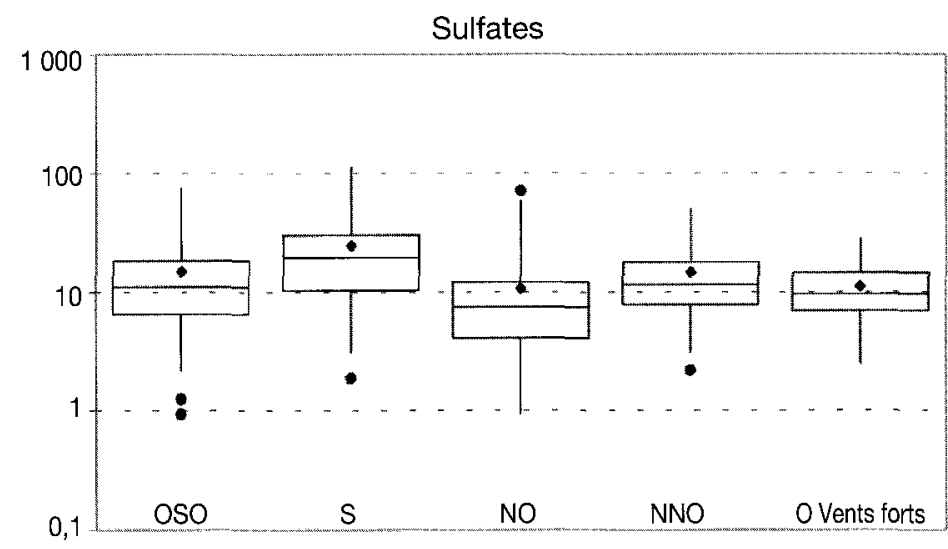

Figure 13 Boîtes à moustaches des concentrations en ions sulfates en $\mu \mathrm{mole} \cdot \mathrm{L}^{-1}$ associées à chaque classe.

Box and whiskers plots for nss-SO $\mathrm{S}_{4}^{2-}$ concentrations $\left(\mu \mathrm{mol} \cdot \mathrm{L}^{-1}\right)$ in precipitation at Iraty and associated with each class of trajectory analyse.

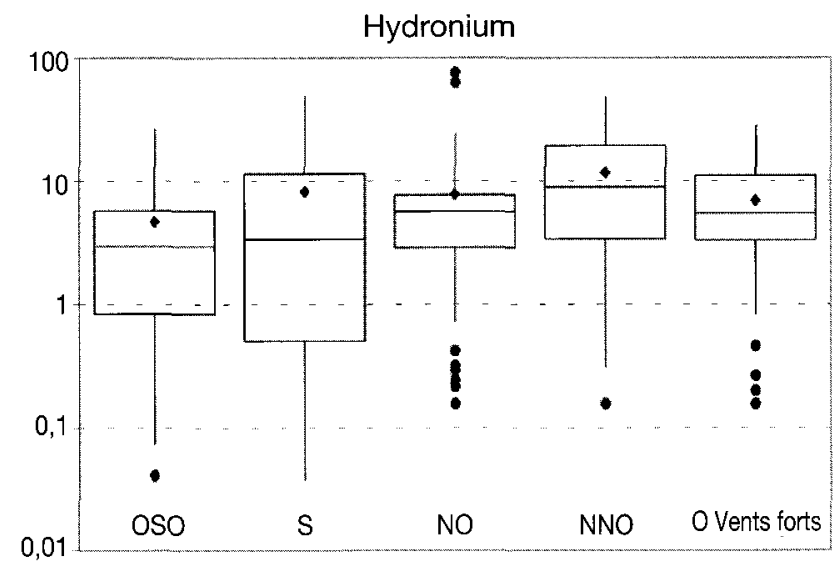

Figure 14 Boîtes à moustaches des concentrations en ions $\mathrm{H}^{+}$en $\mu \mathrm{mole} \cdot \mathrm{L}^{-1}$ associées à chaque classe.

Box and whiskers plots for $\mathrm{H}^{+}$concentrations $\left(\mu \mathrm{mol} \cdot \mathrm{L}^{-1}\right)$ in precipitation at Iraty and associated with each class of trajectory analyse.

fates, nitrates, ammonium et calcium à Iraty devant le secteur OSO (second contribuant) et NNO (troisième contribuant). Ces résultats ont permis de hiérarchiser les zones responsables des plus fortes concentrations à Iraty. Le secteur NNO, aussi en accord avec la carte, s'est montré premier contributeur en ions hydronium. Une analyse en composantes principales suivie d'une rotation varimax nous a permis de révéler que, pour ce secteur, les ions hydronium sont corrélés avec les ions sulfates et nitrates. Ce résultat souligne l'impact des émissions de $\mathrm{SO}_{2}$ et de $\mathrm{NO}_{x}$ de ces régions sur l'acidité des précipitations. II montre, malgré les faibles niveaux de sulfates et nitrates attribués à ces zones, que les émissions de $\mathrm{SO}_{2}$ et $\mathrm{NO}_{x}$ sont bien les responsables de l'acidité. 
D'autre part, la faible corrélation avec les ions ammonium dans cette composante montre que la présence d'aérosols d'ammonium et de $\mathrm{NH}_{3}$ n'a pas neutralisé la totalité des composés soufrés et azotés acides.

Pour le secteur NO (principalement l'océan Atlantique), nous observons aussi une corrélation entre les ions $\mathrm{H}^{+}$et $\mathrm{SO}_{4}^{2-}$. Nous pouvons donc nous demander si c'est l'impact de l'oxydation du DiMéthylSulfide émis par l'océan - ce composé soufré est très rapidement oxydé en $\mathrm{SO}_{2}(\mathrm{YIN}$ et al., 1990) et est donc potentiellement un composé acidifiant - ou de l'impact du $\mathrm{SO}_{2}$ d'origine anthropique émis au niveau de l'océan (transport maritime, par exemple) ou transporté sur de très longues distances depuis l'Amérique. Des faibles quantités de polluants (3\%) seraient transportées d'Amérique jusqu'en Europe (BARRETT et al., 1995).

L'ACP réalisée sur les concentrations associées au secteur Sud, secteur montrant de relativement fortes concentrations à la fois en sulfates, nitrates, ammonium et calcium révèle que ces derniers constituent des composantes principales différentes et donc a priori des sources différentes.

Nous trouvons les associations suivantes:

- ions $\mathrm{NH}_{4}^{+}$et $\mathrm{K}^{+}$, indiquant une origine agricole ;

- ions $\mathrm{NO}_{3}^{-}$et $\mathrm{SO}_{4}^{2-}$, une origine anthropique ;

- ions $\mathrm{Mg}^{2+}$ et $\mathrm{Ca}^{2+}$, une origine crustale.

Enfin, l'ACP montre qu'aucun secteur ne révèle de fortes associations entre les ions sulfates, nitrates et ammonium, comme pour le Morvan. Les coincidences observées sur les cartes indiqueraient seulement des émissions proches géographiquement. $\mathrm{H}_{2} \mathrm{SO}_{4}$ et $\mathrm{HNO}_{3}$ ne seraient pas préférentiellement neutralisés par $\mathrm{NH}_{3}$.

\subsection{Le Casset}

Les cartes suivantes (figures 15 à 19) présentent les résultats obtenus en appliquant la technique du Bootstrapping aux données avant de calculer les champs de concentrations à l'aide de la méthode de SEIBERT et al. Contrairement au Morvan et à Iraty, la carte des concentrations modélisées de sodium ne définit pas l'océan Atlantique comme premier contributeur. De fortes concentrations sont attribuées à l'Afrique du Nord. II est à noter que les concentrations de sodium mesurées au Casset sont globalement très faibles. Néanmoins, la courbe du sodium en fonction du chlore montre une pente de 0,95 (pour une ordonnée à l'origine nulle et un coefficient de corrélation au carré de 0,97), proche du rapport entre le sodium et le chlore de l'eau de mer, égale à 0,86 selon MILLERO (1974), et identique à celui d'Iraty (droite de pente de 0,95 , ordonnée à l'origine nulle et coefficient de corrélation au carré de $0,97)$. Cette très forte association entre ces deux ions montre que les origines du sodium seraient bien océaniques. Ceci démontre un faible enrichissement du sodium par rapport au chlore sur ces deux sites. Cet enrichissement peut suggérer un faible apport crustal. En raison des très faibles niveaux de sodium mesurés au Casset, les cartes de concentrations modélisées en $\mathrm{SO}_{4}^{2-}$ et nss$\mathrm{SO}_{4}^{2-} \mathrm{d}^{\prime}$ une part et en $\mathrm{Ca}^{2+}$ et nss-Ca ${ }^{2+}$ d'autre part, sont identiques. Les cartes de concentrations modélisées en sulfates, nitrates et ammonium montrent deux zones pour lesquelles de plus fortes concentrations ont été attribuées (1) 


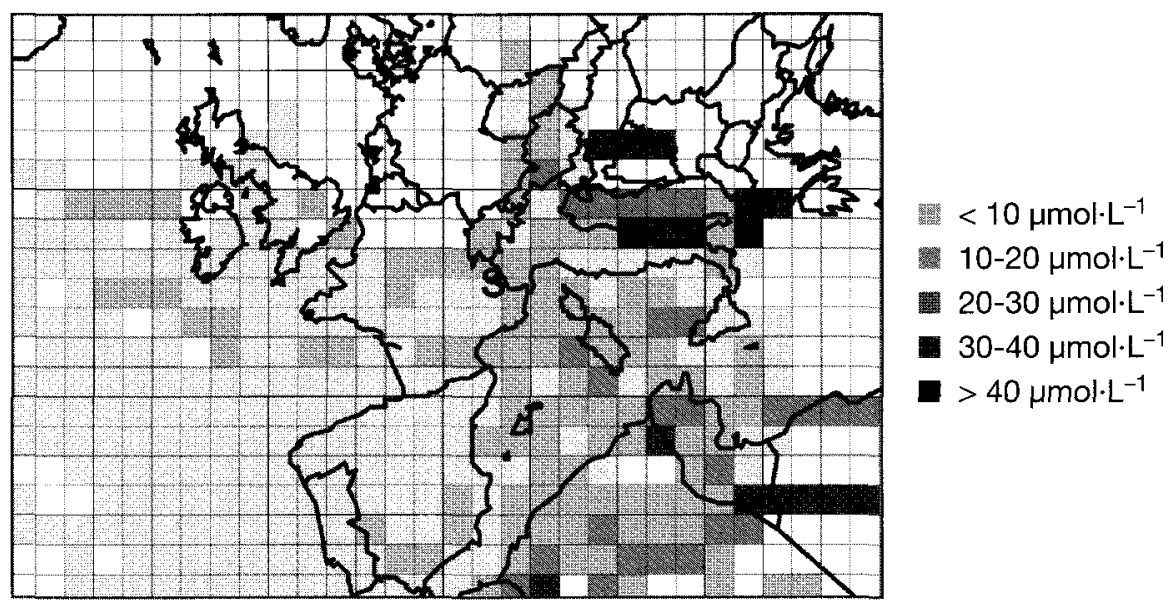

Figure 15 Concentrations modélisées de sulfates mesurés dans les pluies collectées au Casset (1997, juillet 2000)

Average Casset $\mathrm{SO}_{4}^{2-}$ concentrations in precipitation (1997-Jul 2000) according to the origins of air masses.

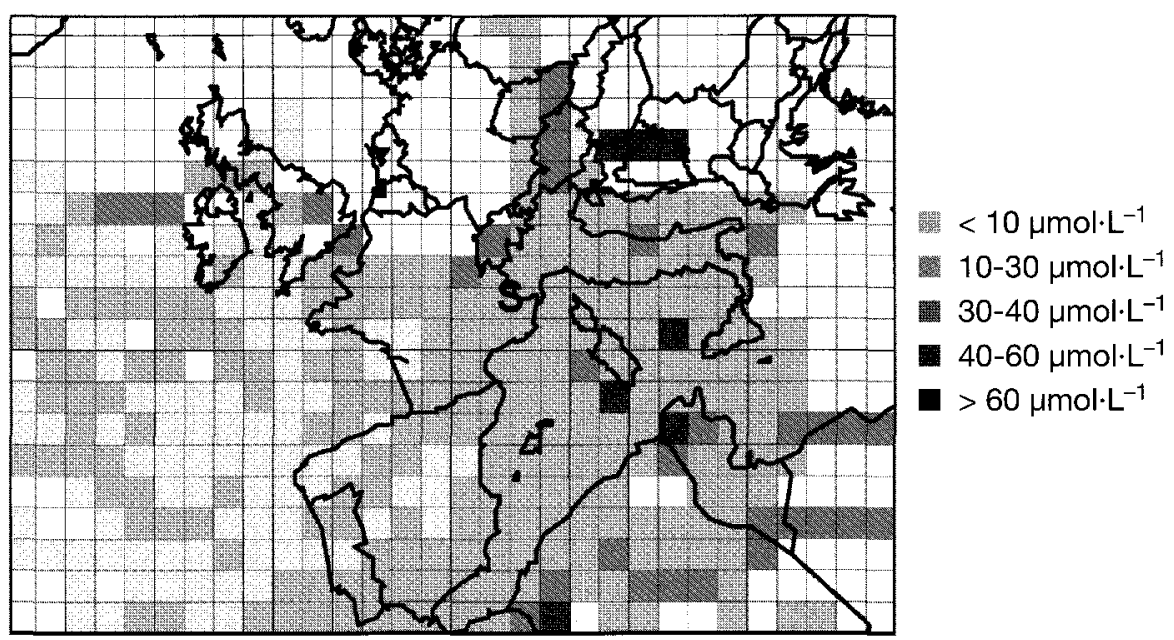

Figure 16 Concentrations modélisées de nitrates mesurés dans les pluies collectées au Casset (1997, juillet 2000).

Average Casset $\mathrm{NO}_{3}^{-}$concentrations in precipitation (1997-Jul 2000) according to the origins of air masses.

I'Afrique, (2) I'Italie/l'ex-Yougoslavie/la mer Adriatique. En comparant avec les cartes des émissions de $\mathrm{SO}_{2}$ et de $\mathrm{NO}_{x}$, la seconde zone définie est effectivement proche de régions fortement émettrices. La précision sur l'Italie n'est néanmoins pas très bonne.

Une fois de plus la carte des concentrations modélisées en ions ammonium s'accorde plus avec les cartes de concentrations modélisées en ions sulfates et nitrates qu'avec la carte des émissions d'ammoniac. 


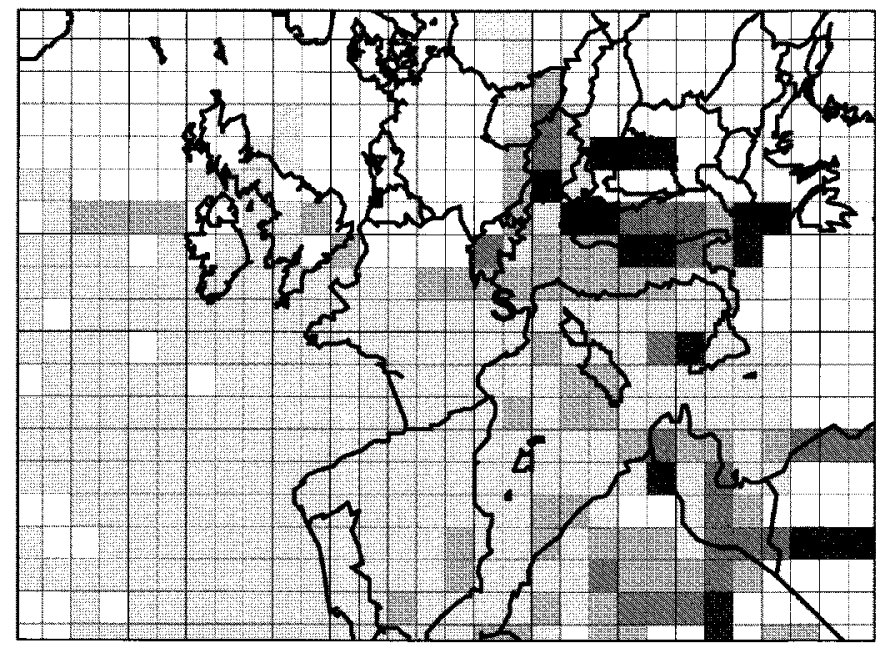

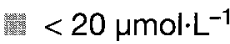

20-40 $\mu \mathrm{mol} \cdot \mathrm{L}^{-1}$

10-50 $\mu \mathrm{mol} \cdot \mathrm{L}^{-1}$

50-80 $\mu \mathrm{mol} \cdot \mathrm{L}^{-1}$

口 $>80 \mu \mathrm{mol} \cdot \mathrm{L}^{-1}$

Figure 17 Concentrations modélisées d'ammonium mesurées dans les pluies collectées au Casset (1997, juillet 2000).

Average Casset $\mathrm{NH}_{4}^{+}$concentrations in precipitation (1997-Jul 2000) according to the origins of air masses.

La carte des concentrations modélisées de calcium (figure 18) montre clairement que les fortes concentrations en calcium mesurées au Casset sont la conséquence du transport atmosphérique de poussières crustales contenant de la calcite depuis l'Afrique du nord. Une précédente étude avait déjà montré que les poussières sahariennes sont transportées jusque dans les Alpes (MAUPETIT et DELMAS, 1994).

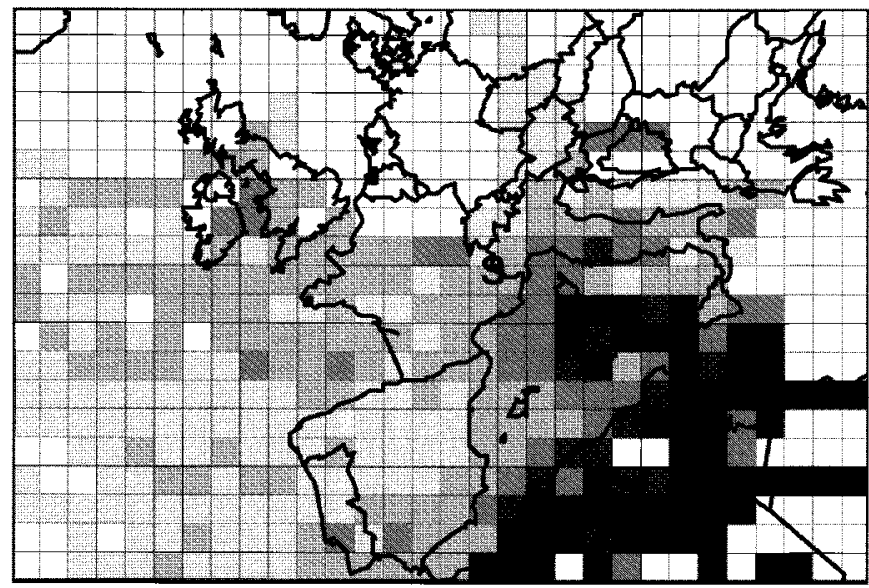

$<10 \mu \mathrm{mol} \cdot \mathrm{L}^{-1}$

$10-20 \mu \mathrm{mol} \cdot \mathrm{L}^{-1}$

20-30 $\mu \mathrm{mol} \cdot \mathrm{L}^{-1}$

30-40 $\mu \mathrm{mol} \cdot \mathrm{L}^{-1}$

- $>40 \mu \mathrm{mol} \cdot \mathrm{L}^{-1}$

Figure 18 Concentrations modélisées de calcium mesurées dans les pluies collectées au Casset (1997, juillet 2000).

Average Casset $\mathrm{Ca}^{2+}$ concentrations in precipitation (1997-Jul 2000) according to the origins of air masses. 
Les $\mathrm{pH}$ (figure 19) les plus faibles sont associés à la seconde zone présentant les concentrations les plus importantes en ions sulfates, nitrates et ammonium suggérant ainsi que le $\mathrm{SO}_{2}$ et les $\mathrm{NO}_{x}$ n'ont pas totalement été neutralisés par l'ammoniac. Les plus forts $\mathrm{pH}$ sont associés avec l'Afrique et le bassin méditerranéen, régions montrant les fortes concentrations modélisées en calcium et suggérant, une fois de plus, l'impact de la calcite $\mathrm{CaCO}_{3}$.

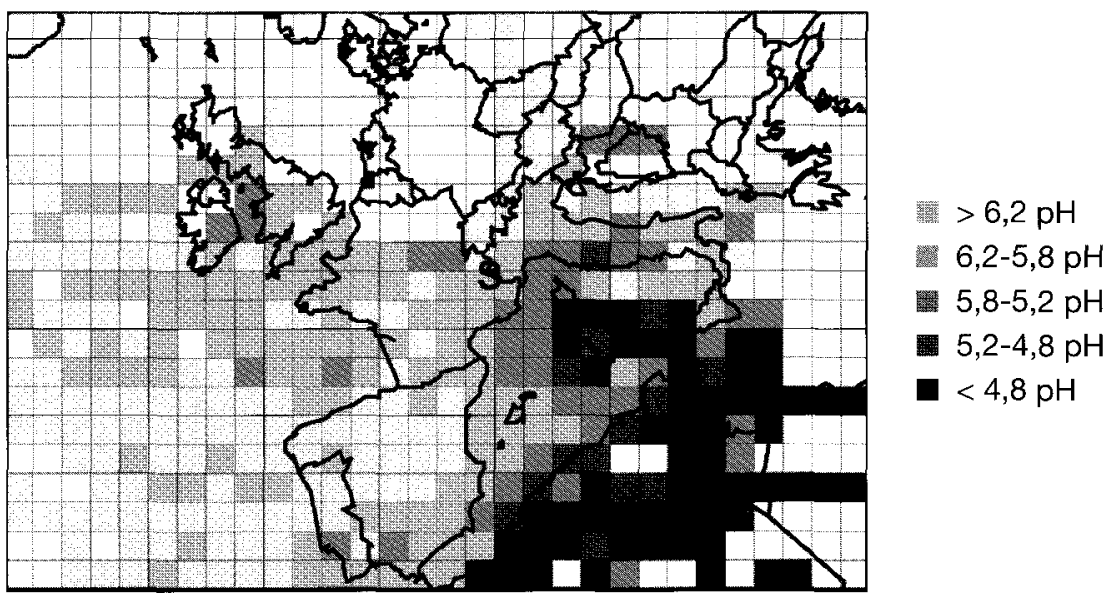

Figure 19 Concentrations modélisées en $\mathrm{H}^{+}$, exprimé en $\mathrm{pH}$, mesurés dans les pluies collectées au Casset (1997, juillet 2000).

Average Casset $\mathrm{H}^{+}(\mathrm{pH})$ concentrations in precipitation (1997-Jul 2000) according to the origins of air masses.

Quatre classes de rétrotrajectoires ont été déterminées à l'aide à l'aide de la classification par Nuées dynamique (figure 20). Deux classes correspondent à des vents d'ouest et représentent à elles deux, 34,7 \% des événements. Deux classes correspondent à des vents de Dud, une de SSO (43,3\% des événements) et une de SE (22\% des événements).

Les concentrations associées à chacun de ces secteurs sont représentées à l'aide de boîtes à moustaches (figures 21 et 22) pour les sulfates et $\mathrm{H}^{+}$.

L'ensemble des résultats est en accord avec les cartes de concentrations modélisées. Les résultats montrent que le secteur SE est le premier contributeur en ions sulfates, nitrates, ammonium et calcium au Casset devant le secteur SSO. De plus faibles concentrations en ions hydronium sont aussi associées avec ce secteur.

L'ACP a montré, pour le secteur $\mathrm{SE}$, que les ions $\mathrm{H}^{+}$sont anticorrélés aux ions $\mathrm{Ca}^{2+}$ et $\mathrm{Mg}^{2+}$, confirmant que ces deux ions proviennent des espèces terrigènes basiques $\mathrm{CaCO}_{3}$ et $\mathrm{MgCO}_{3}$. Pour l'ensemble des secteurs, nous retrouvons l'association entre les ions sulfates, nitrates et ammonium observée pour le Morvan. En ce qui concerne le secteur SSO, les ions $\mathrm{Ca}^{2+}$ et $\mathrm{Mg}^{2+}$ ne sont pas anticorrélés aux ions $\mathrm{H}^{+}$, mais en revanche, sont faiblement corrélés aux ions $\mathrm{SO}_{4}{ }^{2-}$. Nous pouvons supposer qu'il y a eu réaction entre les carbonates et le dioxyde de soufre et/ou $\mathrm{H}_{2} \mathrm{SO}_{4}$. 


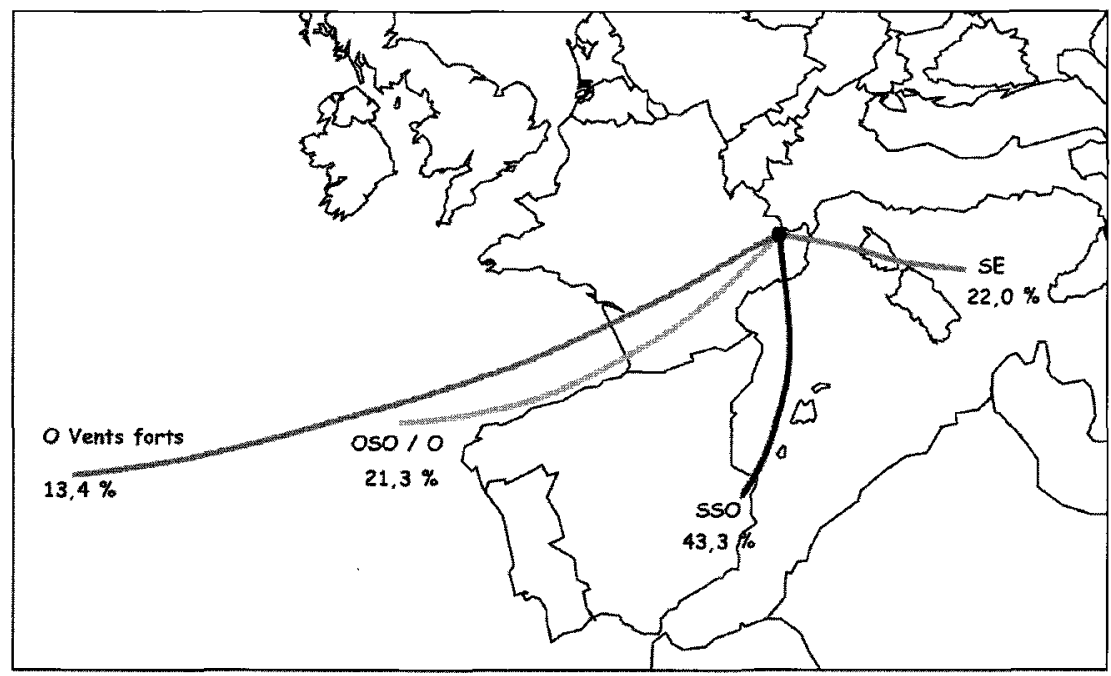

Figure $\mathbf{2 0}$ Centres finaux des classes de rétrotrajectoires associées aux événements pluvieux collectés au Casset pour la période janvier 1997juil. 2000.

$K-M e a n s$ clustering analysis associated with the backtrajectories of rainfall events at Casset (1997-July 2000).

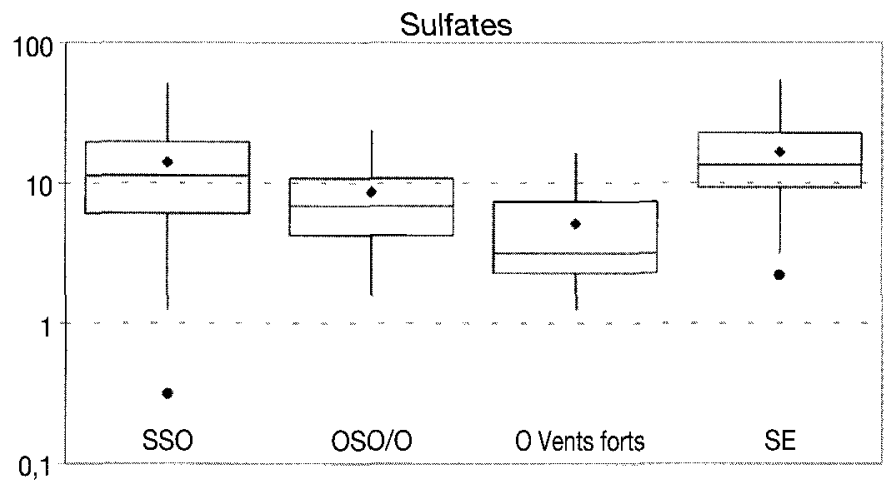

Figure 21 Boîtes à moustaches des concentrations en ions sulfates en $\mu$ mole $\cdot L^{-1}$ associées à chaque classe de secteur prédominant.

Box and whiskers plots for nss-SO $\mathrm{SO}_{4}^{2-}$ concentrations $\left(\mu \mathrm{mol} \cdot \mathrm{L}^{-1}\right)$ in precipitation at Casset and associated with each class of trajectory analyse.

Les résultats présentés pour le Casset ne portent que sur les pluies et donc, sur une partie des précipitations. Il est attendu que la même étude, réalisée avec les précipitations solides, donne des résultats différents du fait, d'une part, de la chimie particulière des neiges et d'autre part, des variations saisonnières des courants atmosphériques responsables des précipitations. Nous avions vu que les variations des courants atmosphériques étaient, au moins en partie, responsables des variations saisonnières des concentrations mesurées dans les précipitations du Morvan (CHARRON et al., 2000). 
Hydronium

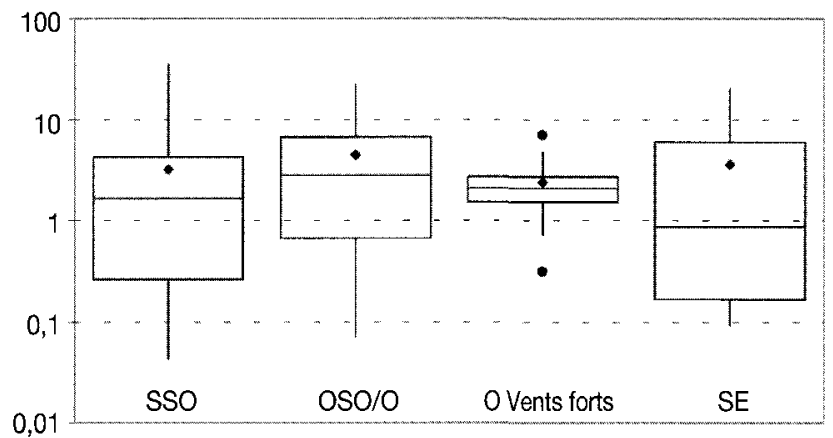

Figure 22 Boîtes à moustaches des concentrations en ions $\mathrm{H}^{+}$en $\mu \mathrm{mole} \cdot \mathrm{L}^{-1}$ associées à chaque classe de secteur prédominant.

Box and whiskers plots for $\mathrm{H}^{+}$concentrations $\left(\mu \mathrm{mol} \cdot \mathrm{L}^{-1}\right)$ in precipitation at Casset and associated with each class of trajectory analyse.

\section{6 - CONCLUSION}

Comme il avait été montré dans l'étude de CHARRON (1999) pour le $\mathrm{SO}_{2}$ et le sulfate particulaire, les résultats relatifs aux précipitations ont montré que les trois sites sont différemment influencés. Les résultats obtenus pour le site du Morvan ont montré l'influence des fortes émissions de $\mathrm{SO}_{2}$ d'Europe centrale et des fortes émissions de $\mathrm{NO}_{x}$ de la zone fortement peuplée située du nord de la France au nord de l'Allemagne. Ces deux zones ne semblent pas influencer le Casset et peu Iraty. Seule la zone fortement peuplée, émettrice de $\mathrm{NO}_{x}$ et assez fortement émettrice de $\mathrm{SO}_{2}$, semble être responsable des pluies de $\mathrm{pH}$ acides à Iraty. L'étude a révélé que les émissions de $\mathrm{SO}_{2}$ et de $\mathrm{NO}_{\mathrm{x}}$ sont les responsables de l'acidité des précipitations.

Iraty et le Casset semblent très influencées par les émissions anthropiques d'Afrique du Nord. Pour ces deux stations, on enregistre les niveaux les plus élevés en $\mathrm{SO}_{4}^{2-}, \mathrm{NO}_{3}^{-}$et $\mathrm{NH}_{4}^{+}$lorsque les masses d'air proviennent d'Afrique du nord. Ces niveaux de concentrations restent néanmoins beaucoup plus faibles que les niveaux de concentrations mesurés dans les flux d'est et de NNO arrivant au Morvan. Ils sont de plus équivalents à ceux mesurées dans les flux de SO arrivant au Morvan. Les masses d'air en provenance d'Afrique du nord, conduisent aussi à de fortes valeurs de $\mathrm{pH}$ et à de fortes concentrations en ions $\mathrm{Ca}^{2+}$ sur les trois sites. En revanche, cette fois-ci Iraty et le Casset semblent bénéficier d'un impact plus important. Une association nette entre la diminution des ions $\mathrm{H}^{+}$et l'augmentation des ions $\mathrm{Ca}^{2+}$ est révélée. Les vents en provenance du sud sont plus fréquents sur ces deux sites. Une relation entre les ions $\mathrm{SO}_{4}^{2-}, \mathrm{NO}_{3}^{-}$et $\mathrm{NH}_{4}^{+}$a été observé pour le Casset et le Morvan. Cette observation suggère le transport d'aérosols d'ammonium type $\mathrm{NH}_{4} \mathrm{HSO}_{4}$, $\left(\mathrm{NH}_{4}\right)_{2} \mathrm{SO}_{4}$ et $\mathrm{NH}_{4} \mathrm{NO}_{3}$. Ces derniers seraient transportés sur de longues distances contrairement à $\mathrm{NH}_{3}$ qui se dépose rapidement à proximité des sources (KITTO et HARRISON, 1992 ; HOV et HJOLLO, 1994). À Iraty, les ions $\mathrm{SO}_{4}^{2-}$ et $\mathrm{NO}_{3}^{-}$ ne semblent pas être préférentiellement liés aux ions $\mathrm{NH}_{4}^{+}$. 
Cette étude pourra être étendue à d'autres régions françaises où la climatologie et les influences des sources d'émission sont différentes mais aussi à d'autres types de pollution gazeuse ou particulaire.

\section{RÉFÉRENCES BIBLIOGRAPHIQUES}

ASHBAUGH L.L., MALM W.C., SADEH W.Z., 1985. A residence time probability analysis of sulfur concentrations at Grand Canyon national park., Atmospheric Environment, 19, 1263-1270.

BARRETT K.O., SELAND A., FOSS A., MYLONA S., SANDNES H., STYVE H., TARRASON L., 1995. European transboundary acidifying air pollution, Report of Meteorological Synthesizing CentreWest, The Norwegian Meteorological Institute, $n^{\circ} 1 / 95$, Oslo, Norway.

CHARRON A., 1999. Caractérisation des retombées atmosphériques acides en zones rurales : étude des relations sources-récepteur et des moyens de mesure adaptés, Thèse de Doctorat, Université de Compiègne.

CHARRON A., PLAISANCE H., SAUVAGE S., CODDEVILLE P., GALLOO JC, GUILLERMO R.A., 2000. Study of the source-receptor relationships influencing the acidity of precipitation collected at a rural site in France. Atmospheric Environment, 34, 3665-3674.

COUDE-GAUSSEN G., ROGNON P., BERGAMETTI G., GOMES L., STRAUSS B., GROS J.M., COUSTUMER M.N.L., 1987. Saharan dust on Fuerteventura island (Canaries): chemical and mineralogical characteristics, air mass trajectories, and probable sources, Journal of Geophysical Research, 92, 9753-9771.

ESTIENNE P., GODARD A., 1970. Climatologie, Armand Colin, Paris.

FANGMEIER A., HADWIGER-FANGMEIER A., EERDEN L.V.D., JÄGER H.-J., 1994. Effects of atmospheric ammonia on vegetation-a review, Environmental Pollution, 86, 43-82.

GALLOWAY J.N., LIKENS G.E., EDGERTON E.S., 1976. Acid precipitation in the northeastern United States: $\mathrm{pH}$ and acidity, Science, 194, 722-724.
HOPKE P.K., LI C.L., CISZEK W., LANDSBERGER S., 1995. The use of bootstrapping to estimate conditional probability fields for source locations of airborne pollutants, Chemometrics an Intelligent Laboratory Systems, 30, 69-79.

HOV O., HJOLLO B.A., 1994. Transport distance of ammonia and ammonium in Northern Europe. 2. Its relation to emissions of $\mathrm{SO}_{2}$ and $\mathrm{NO}_{x}$, Journal of Geophysical Research, 99, 18749-18755.

KITTO A.-B.N., HARRISON R.M., 1992. Processes affecting concentrations of aerosol strong acidity at sites in eastern England, Atmospheric Environment, 26A, 2389-2399.

LEE D.S., KINGDON R.D., PACYNA J.M. BOUWMAN A.F., TEGEN I., 1999 Modelling base cations in Europe sources, transport and deposition of calcium, Atmospheric Environment, 33 2241-2256.

LOSNO R., BERGAMETTI G., CARLIER P., MOUVIER G., 1991. Major ions in marine rainwater with attention to sources of alkaline and acidic species, Atmospheric Environment, 25A, 763-770.

LOYE-PILOT M.D., MORELLI J., 1988. Fluctuations of ionic composition of precipitations collected in Corsica related to changes in the origins of incoming aerosols, Journal of Aerosol Science, 19, 577-585.

MARQUARDT W., IHLE P., 1988. Acidic and alkaline precipitation components in the mesoscale range under the aspect of meteorological factors and the emissions, Atmospheric Environment, 22, 2707-2713.

MAUPETI, F., DELMAS R.J., 1994. Snow chemistry of high altitude glaciers in the French Alps, Tellus, 46B, 304-324.

MIHALOPOULOS N., STEPHANOU E. KANAKIDOU M., PILITSIDIS S., Tropos- 
pheric aerosol ionic composition in the Eastern Mediterranean region, Tellus, 49B, 314-326.

MILLER J.M., MOODY J.L., HARRIS J.M., GAUDRY A., 1993. A 10-year trajectory flow climatology for Amsterdam Island, 1980-1989, Atmospheric Environment, 27A, 1909-1916.

MILLERO F.J., 1974. Physical chemistry of seawater, Annual Reviews of Earth and Planetary Science, 2, 101-150.

MOODY J.L., SAMSON P.J., 1989. The influence of atmospheric transport on precipitation chemistry at two sites in the midwestern United States. Atmospheric Environment, 23, 2117-2132.

MUNGER J.W., 1982. Chemistry of atmospheric precipitation in the North Central United States: influence of sulfate, nitrate, ammonia, and calcareous soil particulates, Atmospheric Environment, 16, 1633-1645.

SEIBERT P., KROMP-KOLB H., BALTENSPERGER U., JOST D.T., SCHWIKOWSKI M., KASPER A., PUXBAUM H., 1994. Trajectory analysis of aerosol measurements at high alpine sites, Proc. of EUROTRAC Symposium'94, ed. P.M. Borrell, P. Borrell, T. Cvitas, W. Seiler, Academic Publishing bv, The Hague, GarmishPartenkirchen, Germany, 689-693.
SEINFELD J.H., PANDIS S.N., 1996. Atmospheric chemistry and physics from air pollution to climate change, New York.

SEQUEIRA R., 1993. On the large-scale impact of arid dust on precipitation chemistry of the continental northern hemisphere, Atmospheric Environment, 27A, 1553-1565.

SIROIS A., BOTTENHEIM J.W., 1995. Use of backward trajectories to interpret the 5year record of PAN and $\mathrm{O}_{3}$ ambient air concentrations at Kejimkujik National Park, Nova Scotia, Journal of Geophysical Research, 100, 2867-2881.

STOHL A., 1996. Trajectory statistics - A new method to establish source -receptor relationships of air pollutants and its application to the transport of particulate sulfate in Europe, Atmospheric Environment, 30, 579-587.

VOLDNER E.C., OLSON M.P., OIKAWA K.K., LOISELLE M., 1981. Comparison between measured and computed concentrations of sulfur compounds measured in eastern North America, $J$. Geophys. Res., 86, 5334-5346.

YIN F., GROSJEAN D., FLAGAN R.C., SEINFELD J.H., 1990. Photooxidation of dimethyl sulfide and dimethyl disulfide. II: Mechanism evaluation, Journal of Atmospheric Chemistry, 11, 365-399. 